\title{
Semi-supervised learning for somatic variant calling and peptide identification in personalized cancer immunotherapy
}

\author{
Elham Sherafat, Jordan Force and Ion I. Măndoiu* (1)
}

From 8th Workshop on Computational Advances in Molecular Epidemiology (CAME 2019) Niagara Falls, NY, USA. 07 September 2019

*Correspondence:
ion@engr.uconn.edu
Computer Science
and Engineering
Department, University
of Connecticut, Storrs, CT
06269, USA

06269, USA

\begin{abstract}
Background: Personalized cancer vaccines are emerging as one of the most promising approaches to immunotherapy of advanced cancers. However, only a small proportion of the neoepitopes generated by somatic DNA mutations in cancer cells lead to tumor rejection. Since it is impractical to experimentally assess all candidate neoepitopes prior to vaccination, developing accurate methods for predicting tumorrejection mediating neoepitopes (TRMNs) is critical for enabling routine clinical use of cancer vaccines.
\end{abstract}

Results: In this paper we introduce Positive-unlabeled Learning using AuTOmI (PLATO), a general semi-supervised approach to improving accuracy of model-based classifiers. PLATO generates a set of high confidence positive calls by applying a stringent filter to model-based predictions, then rescores remaining candidates by using positiveunlabeled learning. To achieve robust performance on clinical samples with large patient-to-patient variation, PLATO further integrates AutoML hyper-parameter tuning, classification threshold selection based on spies, and support for bootstrapping.

Conclusions: Experimental results on real datasets demonstrate that PLATO has improved performance compared to model-based approaches for two key steps in TRMN prediction, namely somatic variant calling from exome sequencing data and peptide identification from MS/MS data.

Keywords: Machine learning, Positive-unlabeled learning, Somatic variant calling, Peptide identification, Exome sequencing, Tandem mass-spectrometry

\section{Background}

Personalized cancer vaccines are emerging as a promising alternative to nonspecific treatments such as chemotherapy in the management of advanced cancers $[1,2]$. This approach harnesses the power of the patient's own immune system to attack cells that express immunogenic peptides called neoepitopes. Neoepitopes are generated as a result author(s) and the source, provide a link to the Creative Commons licence, and indicate if changes were made. The images or other third party material in this article are included in the article's Creative Commons licence, unless indicated otherwise in a credit line to the material. If material is not included in the article's Creative Commons licence and your intended use is not permitted by statutory regulation or exceeds the permitted use, you will need to obtain permission directly from the copyright holder. To view a copy of this licence, visit http:// creativecommons.org/licenses/by/4.0/. The Creative Commons Public Domain Dedication waiver (http://creativecommons.org/publi cdomain/zero/1.0/) applies to the data made available in this article, unless otherwise stated in a credit line to the data. 
of somatic DNA mutations that arise in cancer cells, hence making the immune response tumor-specific. However, only a small proportion of the potential neoepitopes lead to tumor rejection [3-6]. Methods for predicting tumor-rejection mediating neoepitopes (TRMNs) are the subject of much active research, including large consortium efforts such as the Tumor Neoantigen Selection Alliance [7].

Existing bioinformatics pipelines for neoepitope and TRMN prediction (e.g., [8-11]) include two main steps: (1) calling tumor-specific somatic variants from matched tumornormal exome or whole-genome sequencing data, and (2) predicting which mutated peptides generated by non-synonymous somatic variants are presented to the immune system by the Major Histocompatibility Complex (MHC) alleles of the patient. Recent experimental work using a mouse tumor model [12] supports the utility of incorporating a third step, which prioritizes for vaccination the mutated peptides detected by tandem mass-spectrometry (MS/MS) in elutions of peptide-MHC complexes recovered from the surface of tumor cells.

Although many bioinformatics tools exist for each of these steps, there is still significant room for improvement. In particular, although many somatic variant callers have been developed based on diverse statistical models, agreement between them remains low $[13,14]$. Key impediments to achieving consistently high accuracy with model-based methods include the large patient-to-patient variation in tumor purity and heterogeneity, sequencing library preparation artifacts, sequencing errors, and data processing errors such as incorrect read alignment. Several machine learning methods for somatic mutation calling have been recently developed to address this challenge [15-22]. However, most of these methods adopt a supervised learning paradigm and generally require large amounts of training data.

In this paper we introduce a novel machine learning approach aimed at increasing the sensitivity of any existing model-based pipeline for somatic variant calling while maintaining a high positive predictive value. To achieve robust performance despite the significant patient-to-patient variation present in clinical samples, we adopt a semisupervised approach that learns salient attributes from the data itself, without a need for prior training datasets. Our approach, referred to as Positive-unlabeled Learning using AuTOml (PLATO), is illustrated in Fig. 1 (see also the flowchart in Fig. 2). PLATO takes as input the list of unfiltered candidate somatic variant calls generated using an existing model-based pipeline along with a subset of highly confident calls obtained by applying stringent thresholds. PLATO adopts a Positive-Unlabeled (PU) learning approach, in which the set of highly confident calls are used as positive examples and the remaining candidate calls are used as unlabeled examples. Real cancer datasets have typical unlabeled:positive ratios of 1000:1 or higher. The vast majority of unlabeled examples are a priori expected to be true negatives (sequencing errors or germline variants). PLATO takes advantage of this skewed distribution to generate likely negative datasets by informed undersampling, i.e., randomly picking points that are furthest from the positive set according to the Gower distance in a space defined by categorical and numerical features such as confidence scores and allele coverage information generated by the model-based pipeline and sequence properties extracted from the genome and alignment files. PLATO then trains a classifier to discriminate between the positive and likely negative examples, and uses this classifier to label remaining data points. 


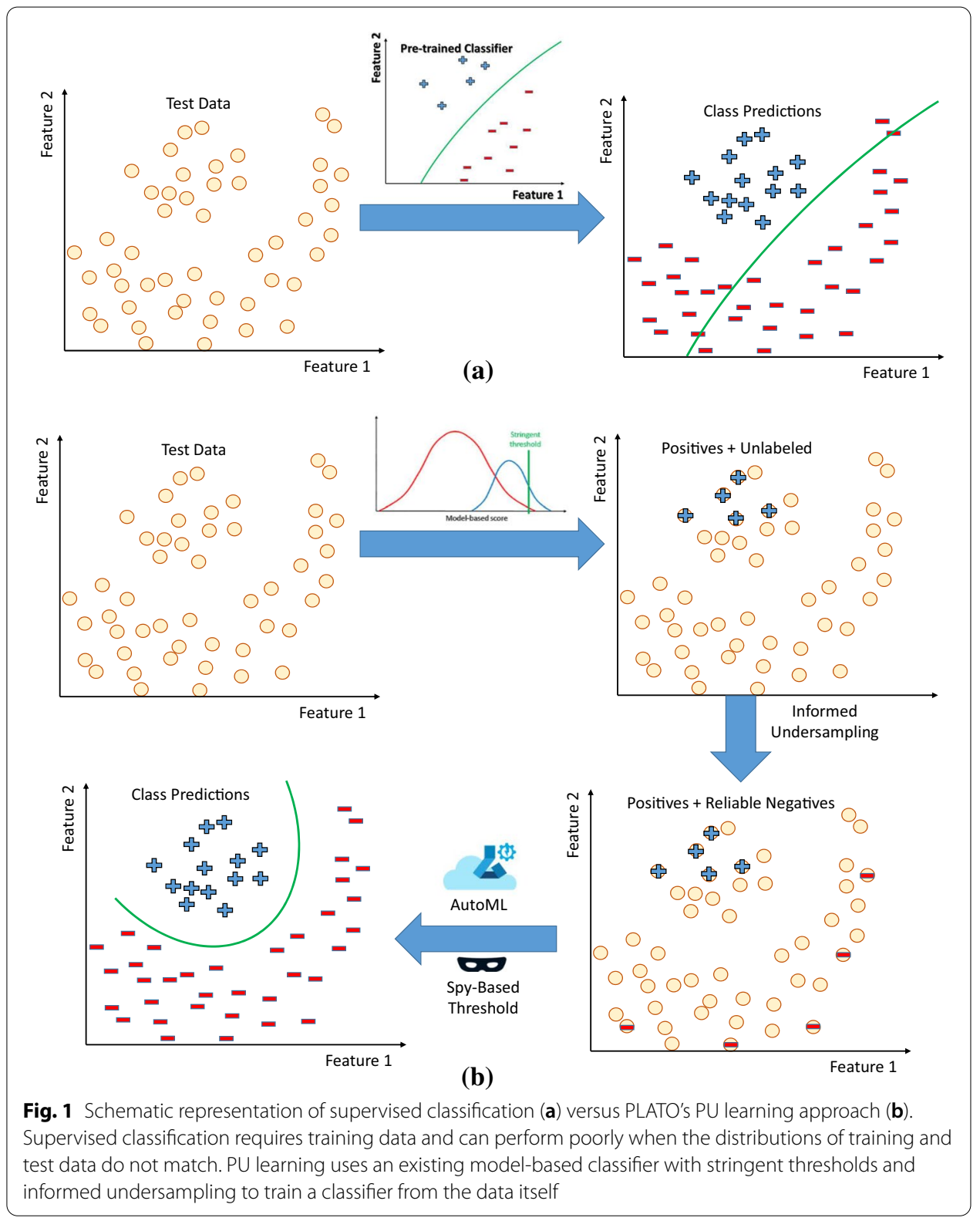

Hyper-parameter tuning is performed by cross-validation using the AutoML service provided by Microsoft Azure. Additionally, PLATO uses a "spy" approach for robust classification threshold selection, and performs a user specified number of bootstraps, reporting only variants with $50 \%$ or higher bootstrap support.

\section{Results}

\section{Somatic variant calling from multi-technology exome sequencing data}

To assess PLATO's accuracy we used matched normal-tumor exome sequencing data generated for four ovarian cancer patients (identified in this article as P1 to P4) using two different sequencing technologies, Illumina and Ion Torrent.The unlabeled set given as input to PLATO was generated using the Consensus Caller Cross-Platform 


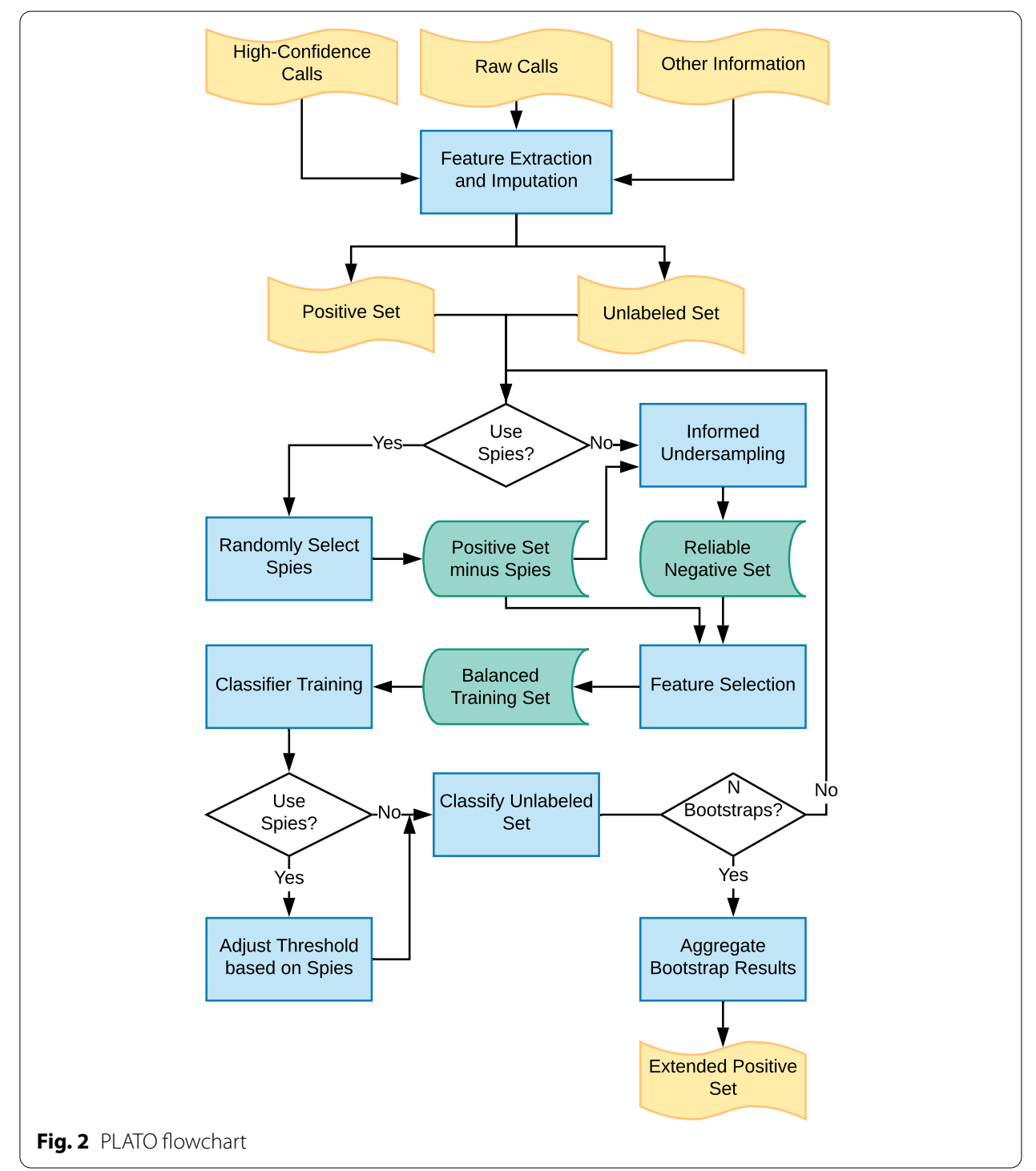

(CCCP) Galaxy tool available as part of the GeNeo immunogenomics toolbox [23]. CCCP incorporates two state-of-the-art somatic mutation callers, SNVQ [24] and Strelka [25], and has the ability to process multi-technology sequencing data. Positive calls were generated by applying the $2 \mathrm{CP}$ filter [26] on the raw output of CCCP. $2 \mathrm{CP}$ requires that at least one of the two callers make a high confidence call from each of the two sequencing technologies. The only exception is when one of the sequencing technologies yields no read coverage, in which case both callers must make confident calls from the reads generated by the complementary technology. The ground truth for a subset of the predicted somatic variants was established by taking the consensus of calls made from high-depth targeted re-sequencing of amplicons generated using the AccessArray system from three or more replicates per patient of both tumor and normal tissue. The first column of Table 1 gives the number of resequenced variants for each patient along with the sizes of the $P$ and $U$ sets. In all cases, the resequenced set included all variants that passed the $2 \mathrm{CP}$ filter and for which AccessArray primers 
Table 1 Variant calling performance on sequencing datasets P1-P4 generated for four ovarian cancer patients

\begin{tabular}{lllrrrrlll}
\hline DataSet & Caller & \# calls & TP & FP & TN & FN & TPR & PPV & F1 \\
\hline P1 & SNVQ & 515 & 48 & 114 & 0 & 0 & 100 & 29.63 & 45.71 \\
P: 65 & Strelka & 435 & 48 & 34 & 80 & 0 & 100 & 58.54 & 73.85 \\
U: 29758 & 2CP & 65 & 41 & 8 & 106 & 7 & 85.42 & 83.67 & 84.54 \\
Reseq: 162 & PLATO & 587 & 42 & 6 & 104 & 10 & 80.77 & 87.5 & 84 \\
P2 & SNVQ & 597 & 147 & 65 & 3 & 2 & 98.66 & 69.34 & 81.44 \\
P: 187 & Strelka & 619 & 149 & 59 & 9 & 0 & 100 & 71.63 & 83.47 \\
U: 31210 & 2CP & 187 & 133 & 39 & 29 & 16 & 89.26 & 77.33 & 82.87 \\
Reseq: 217 & PLATO & 449 & 144 & 43 & 25 & 5 & 96.64 & 77.01 & 85.71 \\
P3 & SNVQ & 629 & 61 & 13 & 8 & 1 & 98.39 & 82.43 & 89.71 \\
P: 76 & Strelka & 306 & 62 & 11 & 10 & 0 & 100 & 84.93 & 91.85 \\
U: 30289 & 2CP & 76 & 57 & 1 & 20 & 5 & 91.94 & 98.28 & 95 \\
Reseq: 83 & PLATO & 429 & 62 & 3 & 18 & 0 & 100 & 95.38 & 97.64 \\
P4 & SNVQ & 482 & 48 & 23 & 87 & 2 & 96 & 67.61 & 79.34 \\
P: 67 & Strelka & 380 & 50 & 94 & 16 & 0 & 100 & 34.72 & 51.55 \\
U: 30176 & 2CP & 67 & 45 & 2 & 108 & 5 & 90 & 95.74 & 92.78 \\
Reseq: 160 & PLATO & 490 & 48 & 7 & 103 & 2 & 96 & 87.27 & 91.43 \\
\hline
\end{tabular}

could be successfully designed using the primer design tool in GeNeo. The resequenced sets also included additional SNVs called using a random forest classifier at varying levels of bootstrap support. For each compared method we computed the number of true positives (TP), false positives (FP), true negatives (TN), and false negatives (FN) relative to the set of variant calls for which the ground truth was available. The reported true positive rate, $T P R:=T P /(T P+F N)$, positive predictive value, $P P V:=T P /(T P+F P)$, and $F 1$ score, $F 1:=2 \cdot T P R \cdot P P V /(T P R+P P V)$, were also computed relative to the ground truth available for each method.

\section{Effect of classification threshold selection and classification algorithm}

The users of PLATO can choose between automatic classification threshold selection based on spies or using the underlying classifier's default threshold (typically 0.5). Also, in principle, the PLATO framework can be used in conjunction with any supervised classification algorithm. AutoML already integrates a wide range of supervised classification methods, dynamically evaluating them on each dataset using a cross-validation approach to avoid over-fitting. However, using AutoML does come with an added computational cost. To see if this added cost is warranted, we compared the AutoML-based implementation of PLATO with a baseline implementation based on random forests.

Figure 3a, b show that, for both the random forest and AutoML implementations, using spies-based classification thresholds yields F1 scores close to and often better than those obtained by using the classifier's default threshold. This holds independently of the bootstrap support required for positive classification. Furthermore, Fig. $3 \mathrm{c}$ shows that, when using spies-based thresholds, the AutoML-based implementation of PLATO has F1 score comparable to or better than those of the random forest implementation at virtually all bootstrap support cutoffs. 


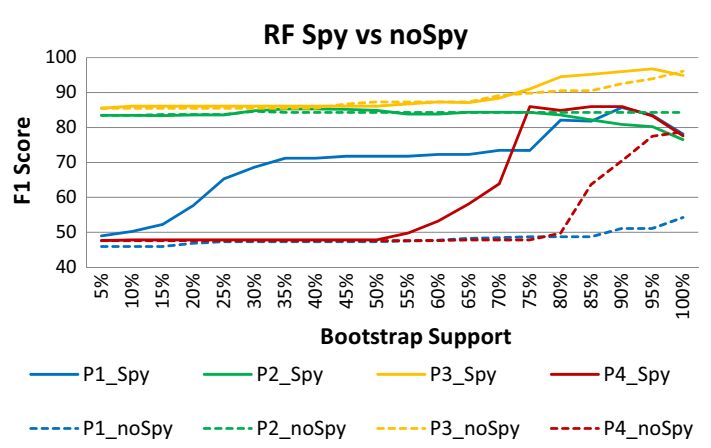

(a)

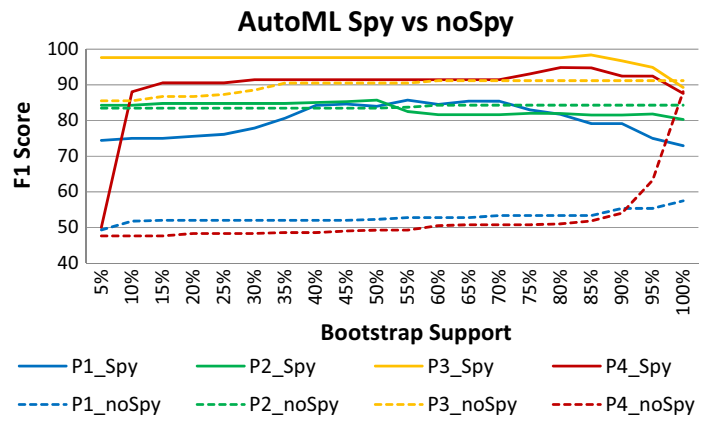

(b)

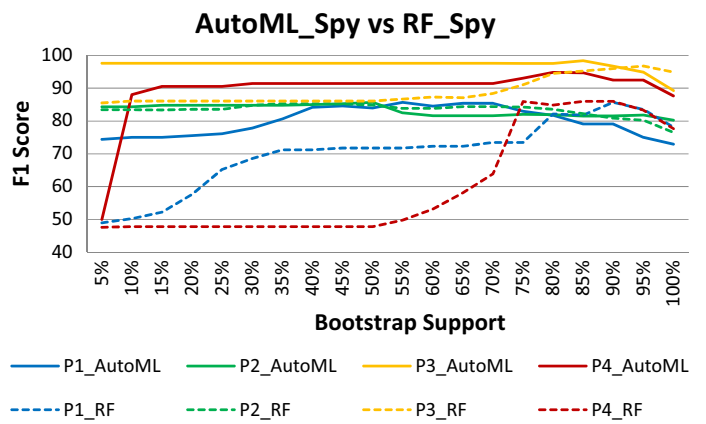

(c)

Fig. 3 F1 scores obtained by running PLATO with $N=20$ bootstraps. a Random forest classification with spies-based classification threshold versus 0.5 default, b AutoML classification with spies versus 0.5 default, and $\mathbf{c}$ AutoML with spies versus random forest with spies. P1-P4 denote the sequencing datasets generated for four different ovarian cancer patients

\section{Comparison with model-based callers}

Table 1 gives detailed accuracy results on the four ovarian cancer datasets, comparing PLATO with model-based callers SNVQ [24] and Strelka [25], as well as the 2CP filter of CCCP [26]. PLATO results in this table were obtained by using AutoML as classifier, spies-based classification threshold selection, $N=20$ bootstraps, and $50 \%$ bootstrap support. On all four datasets, the F1 score of PLATO is comparable to or better than that of $2 \mathrm{CP}$, which in turn is comparable to or better than that of SNVQ and Strelka. Unlike SNVQ and Strelka, PLATO always retains a high PPV, comparable to or better than that of 2CP. This is important, since PLATO also makes between $2.4 \times$ and $9 \times$ more calls than the very stringent $2 \mathrm{CP}$ filter. Assuming a constant PPV 
this suggests that substantially more SNVs are expected to be confirmed when resequencing candidates called by PLATO on the AccessArray. Figure 4 shows for each caller the expected TP count assuming a constant PPV for up to 480 primer pairs multiplexed on a 48.48 AccessArray IFC. For reference, Fig. 4 also includes dots representing the counts from the actual AccessArray resequencing experiment reported in Table 1.

\section{Feature importance for SNV calling}

Figure $5 \mathrm{a}$ gives the importance reported by AutoML for the top 10 features used for SNV calling, averaged for each dataset over 20 bootstrap runs (for feature descriptions see Additional file 1). Not surprisingly, the top four features are the binary somatic calls made for each sequencing technology (Illumina and Ion Torrent) by the two callers integrated in CCCP (SNVQ and Strelka). Binary calls made by SNVQ from the normal Illumina and Ion Torrent exomes and dbSNP status follow close behind in importance. The variation in feature importance from dataset to dataset is remarkably high, underscoring the need for semi-supervised methods such as PLATO that can adapt to the idiosyncrasies of each dataset. Figure $5 \mathrm{~b}$ gives boxplots of the classification cutoffs selected using the spy approach over the 20 bootstraps runs performed for each of the four datasets. Most likely due to the over-representation of negatives in the list of CCCP candidates, the spy-based cutoffs are always higher than the 0.5 default. Furthermore, the cutoff distributions vary from patient to patient, again underscoring PLATO's ability to adapt to each dataset.

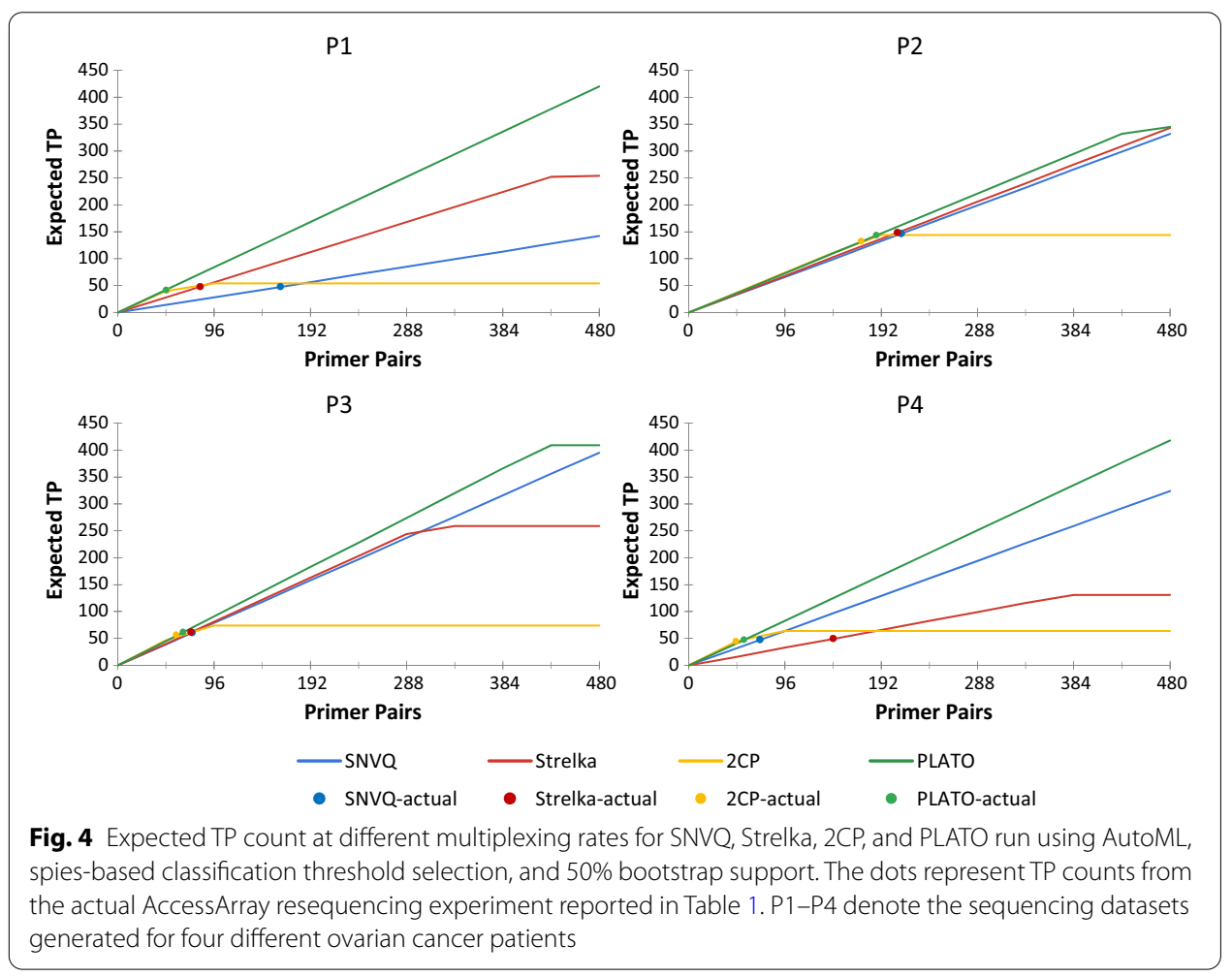




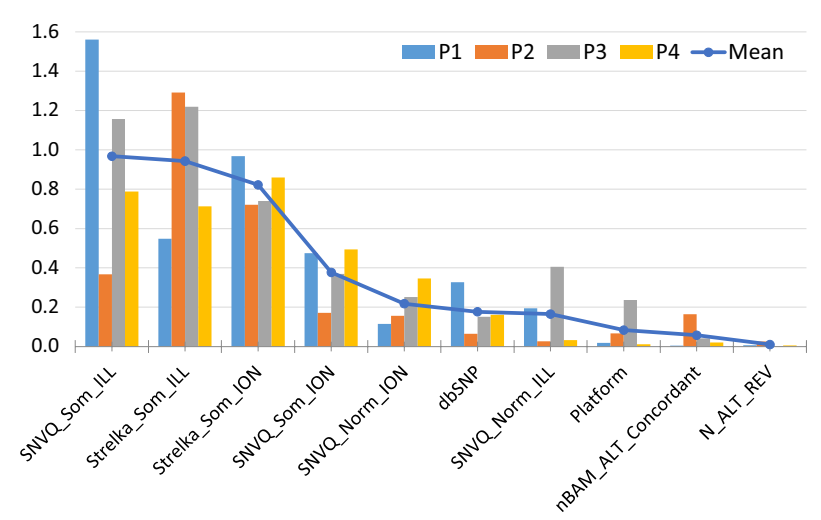

(a)

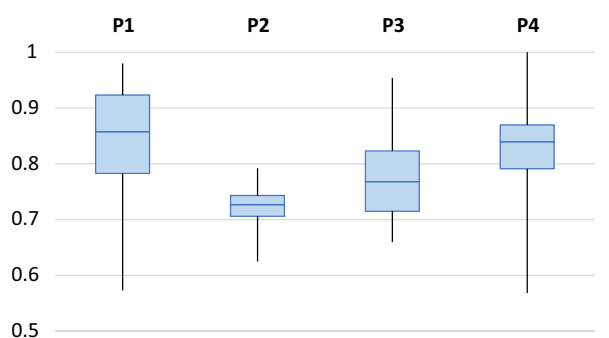

(b)

Fig. 5 Average feature importance for SNV calling (a), and boxplots of the classification cutoffs selected using the spy approach (b) over the 20 bootstraps runs performed for the P1-P4 ovarian cancer datasets

\section{Peptide identification from MS/MS data}

For peptide identification, we evaluated our method on twenty datasets generated by [27] and retrieved from the ProteomeXchange repository using project identifier PXD004894. We retrieved the RAW MS/MS files for five different melanoma patients (identified as mel3, mel4, mel5, mel8, and mel12). For each patient we retrieved four MS/ MS files, corresponding to two biological replicates per patient (p1/p2) and two independent MS/MS runs per replicate (identified by the date of the run, 2014-03-04/201403-05 or 2014-03-06). Table 2 gives the number of peptides identified at a $q$-value cutoff of 0.01 by MS-GF+, Percolator, and PLATO. Although both Percolator and MS-GF+ can compute PSM and Peptide level $q$-values, the $q$-values for all three methods were computed by our implementation of the procedure described in the Methods section to ensure that differences in peptide counts between the different methods are not due to variations in the $q$-value computation method.

For comparison, Table 2 also includes the number of peptides identified in [27] using the MaxQuant search engine with the same FDR cutoff. While we provide these numbers as a baseline, they should be considered with caution, because MaxQuant was used to search a different human proteome database. For MS-GF+ searches we used 20,585 protein sequences retrieved from Uniprot in 2019 (see Additional file 1 for details), while [27] searched a database containing 85,919 protein sequences retrieved in 2014. As shown in Table 2 and visualized as improvement over the MaxQuant baseline in Fig. 6, both PLATO and Percolator significantly outperform MaxQuant and MS-GF+ in terms of the number of peptides identified at $1 \%$ peptide-level FDR. Although their 
Table 2 Number of peptides identified at 1\% FDR from 20 MS/MS datasets generated by [27]

\begin{tabular}{lllll}
\hline Sample ID & Max quant & MS-GF+ & Percolator & PLATO \\
\hline 2014-03-04-mel3p1 & 2533 & 2967 & 3748 & 3898 \\
2014-03-04-mel3p2 & 2770 & 3341 & 4467 & 4412 \\
2014-03-06-mel3p1 & 2441 & 2704 & 3632 & 3681 \\
2014-03-06-mel3p2 & 2594 & 3140 & 4271 & 4157 \\
2014-03-04-mel4p1 & 2634 & 3108 & 3929 & 4073 \\
2014-03-04-mel4p2 & 1765 & 3073 & 4004 & 3757 \\
2014-03-06-mel4p1 & 2401 & 2824 & 3526 & 3682 \\
2014-03-06-mel4p2 & 1745 & 2694 & 3856 & 3954 \\
2014-03-05-mel5p1 & 3010 & 2517 & 3742 & 3923 \\
2014-03-05-mel5p2 & 3342 & 2561 & 4023 & 4127 \\
2014-03-06-mel5p1 & 2934 & 2643 & 3929 & 4059 \\
2014-03-06-mel5p2 & 3060 & 2592 & 4070 & 4014 \\
2014-03-05-mel8p1 & 3375 & 3057 & 4006 & 4297 \\
2014-03-05-mel8p2 & 3764 & 2976 & 4511 & 4556 \\
2014-03-06-mel8p1 & 3331 & 3023 & 4375 & 4454 \\
2014-03-06-mel8p2 & 4139 & 3444 & 4801 & 4839 \\
2014-03-04-mel12p1 & 1948 & 1601 & 2724 & 2870 \\
2014-03-04-mel12p2 & 2013 & 1408 & 2855 & 3121 \\
2014-03-06-mel12p1 & 2004 & 1301 & 3028 & 3005 \\
2014-03-06-mel12p2 & 2628 & 1601 & 2942 & 3356 \\
\hline
\end{tabular}

MHC-bound peptides were eluted from melanoma samples collected from five different patients (identified as mel3, mel4, mel5, mel8, and mel12), with two biological replicates (p1/p2) per patient, each analyzed on two independent MS/MS runs (identified by the date in the sample ID). For each MS/MS dataset, the largest number of identified peptides is typeset in italics

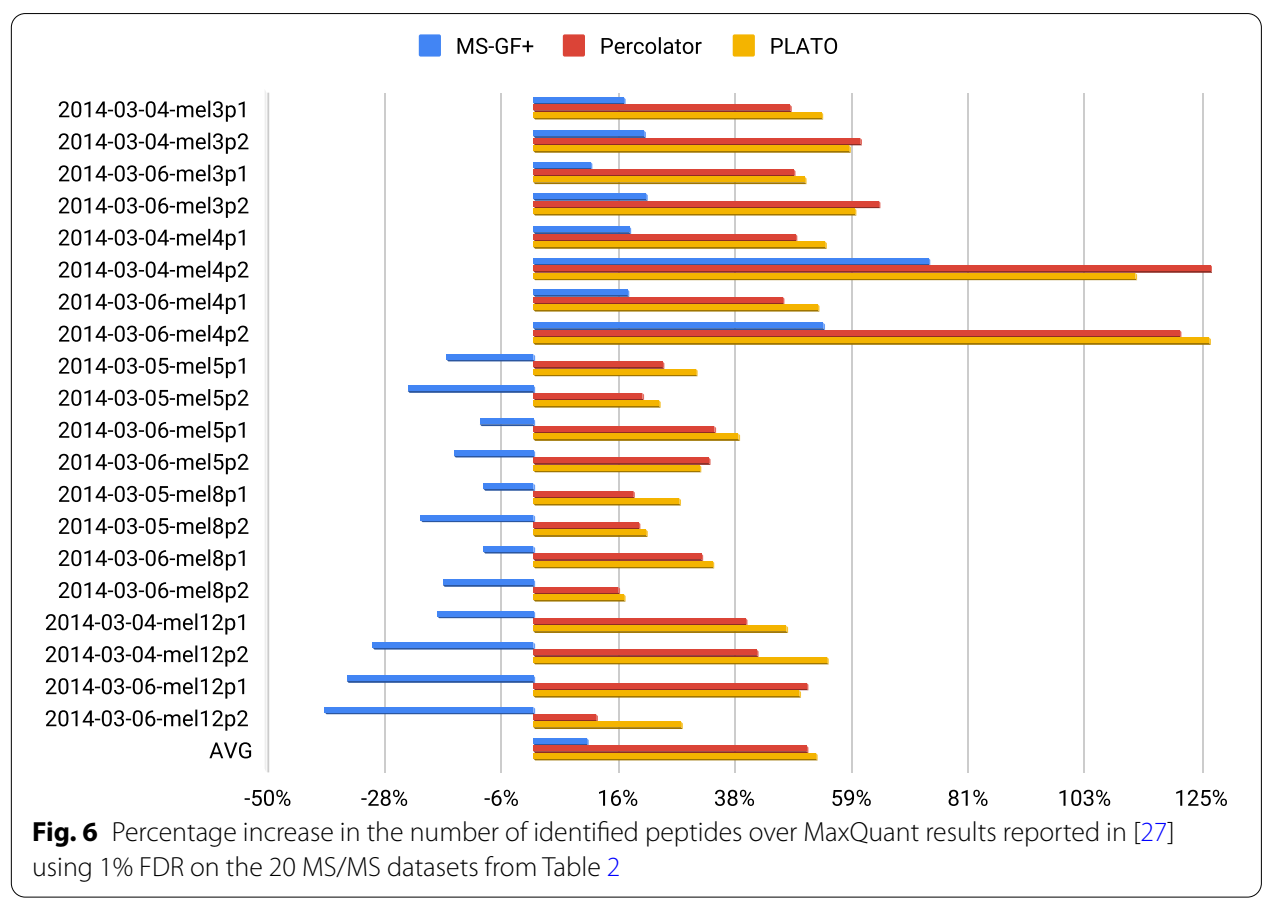


perfomance is comparable, PLATO has a slight edge over Percolator, outperforming it on 15 out of the 20 datasets while being outperformed only 5 times.

\section{Feature importance for peptide identification}

The top 15 features ranked by average AutoML importance are shown in Fig. 7 (for feature descriptions see Additional file 1). The importance score of the lnEValue dominates the scores of the other features by more than one order of magnitude, and has relatively small sample-to-sample variation. Interestingly, the amino acids at known anchor positions for MHC class I binding have relatively low importance scores, most likely due to the fact that clinical MS/MS samples are comprised of peptides presented by up to six distinct MHC class I alleles, each with potentially different anchor position specificities.

\section{Discussion}

Experimental validation results on sequencing data from four ovarian cancer patients demonstrate the effectiveness of PLATO when combined with the existing Consensus Caller Cross-Platform (CCCP) pipeline for somatic variant calling [23]. Since the PU learning framework is broadly applicable, we also applied PLATO to improve the rate of confident peptide identification from tandem mass-spectrometry data. Specifically, we combined PLATO with the open-source MS-GF+ database search engine [28], and used it to rescore peptide-spectrum matches (PSMs) using MS-GF+ features such as the match-score and spectrum charge, along with sequence defined features such as aminoacid composition and context. The use of PLATO increases the number of identified peptides at a fixed false discovery rate (FDR) compared to both model-based database search engines MS-GF+ and MaxQuant as well as the Percolator method, an existing rescoring approach based on support vector machines [29].

We have made available user-friendly web-based tools for peptide identification from MS/MS data by running the MS-GF+ and Percolator algorithms under the "Immunopeptidomics" section of the GeNeo Galaxy toolbox for Genomics Guided Neoepitope Prediction [8]. More information about these tools is provided in Additional file 1. A Python script that can be used to run PLATO on the output files generated by MS-GF+

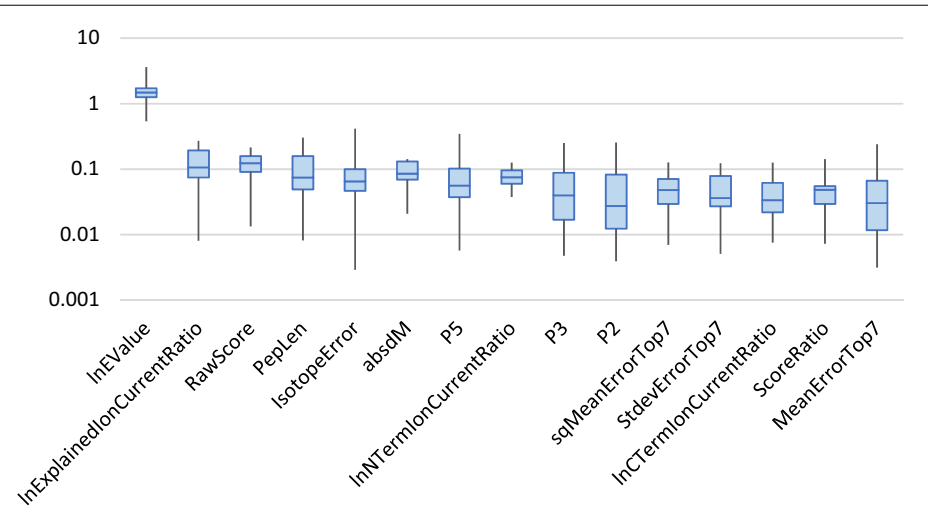

Fig. 7 Boxplots of feature importance values (displayed on a logarithimic scale) for PLATO peptide identification experiments on the 20 MS/MS datasets from Table 2 
is also available at github.com/esherafat/PLATO. Integration of PLATO into the GeNeo toolbox [8] is ongoing.

In future work we plan to assess PLATO's robustness to intra-tumor heterogeneity using large-scale exome sequencing datasets such as [30] and explore further improvements in peptide identification accuracy by incorporating additional features in the PLATO search. Finally, we plan to explore supervised and semi-supervised methods for predicting TRMNs. Improving TRMN prediction accuracy is critical for enabling routine clinical use of cancer vaccines since it is impractical to experimentally assess all candidate neoepitopes prior to vaccination [31].

\section{Conclusion}

In this paper we introduced PLATO, a novel semi-supervised approach to improving accuracy of model-based classifiers. PLATO generates a set of high confidence positive calls by applying a stringent filter to model-based predictions, then rescores remaining candidates by using positive-unlabeled learning. PLATO further integrates AutoML hyper-parameter tuning, classification threshold selection based on spies, and bootstrapping to achieve robust performance on clinical samples with large patientto-patient variation. Although the PU-learning framework implemented by PLATO is broadly applicable, in this paper we focused on its application and evaluation in the context of two problems arising in personalized cancer immunotherapy: somatic variant calling from matched tumor-normal exome sequencing data and peptide identification from immunopeptidomic MS/MS data. This allowed us to leverage the ability to conduct experimental validation of somatic variant calls as part of an ongoing clinical trial and rely on well-established techniques for controlling false discovery rate based on template-decoy competition in the case of peptide identification from MS/MS data. Experimental results on real datasets show improved PLATO performance compared to model-based approaches for both applications.

\section{Methods}

\section{Positive-unlabeled learning}

Semi-supervised learning is used when available training data is a combination of labeled and unlabeled samples. The key idea of semi-supervised learning is to use the unlabeled examples to modify, refine or prioritize the hypotheses derived from the labeled data alone. Positive-unlabeled learning is an important subcategory of semi-supervised learning, where only unlabeled and positive samples are available. One popular technique for PU learning is to predict a set of likely negatives among the unlabeled samples and then apply standard supervised machine learning methods to the set of positives and likely negatives. The PU learning framework implemented in PLATO is illustrated in Fig. 2. Below we detail the key steps of this workflow.

\section{Feature extraction and imputation}

For variant calling, the sets $P$ and $U$ were generated from the output of the Consensus Caller Cross-Platform (CCCP) pipeline [23]. The set of positives was taken to be the set of variants passing the $2 \mathrm{CP}$ filter [26] that comes with the CCCP pipeline, and all other SNV candidates were included in $U$. Both positive and unlabeled samples were 
represented using a total of 110 features, 52 extracted from the output of the CCCP pipeline (see Table S3 in Additional file 1) and 58 generated using SomaticSeq [16] from the BAM files containing Illumina tumor and normal exome alignments (full list included in Additional file 1). This broad range of features included somatic variant calls made by the two somatic variant callers integrated in CCCP (SNVQ [24] and Strelka [25]), the coverage in tumor and normal samples, variant allele frequency, strand bias, membership in the list of common polymorphisms catalogued in the dbSNP database [32], average base and alignment quality, genomic region mappability, etc. The unfiltered output of CCCP includes a large percentage of missing values, typically due to low read coverage from one of the sequencing technologies. To deal with these missing values, prior to performing informed undersampling we removed the samples and features for which more than half of the corresponding entries were missing. Additionally, for the remaining samples we imputed missing features using the rfImpute function implemented by the randomForest CRAN package [33].

For peptide identification from MS/MS data, $P$ and $U$ were generated from the list of best peptide-spectrum matches (PSMs) generated using the MS-GF+ search engine for each spectrum (see Additional file 1 for details). $P$ was taken to be the set of PSMs identified by MS-GF+ at a False Discover Rate (FDR) cutoff of 1\% (as estimated by targetdecoy competition, see below), while $U$ consisted of the remaining PSMs. PLATO was run using 27 features extracted from the MS-GF+ output (see Table S4 in Additional file 1). No imputation was performed for the MS/MS data.

\section{Informed undersampling and feature selection}

In both of our applications (SNV calling from matched tumor-normal sequencing data and peptide identification from tandem mass-spec data) the number of unlabeled samples vastly exceeds the number of labeled positives. For example, cancer datasets have a typical unlabeled:positive ratio of 1000:1 or higher. The vast majority of unlabeled examples are a priori expected to be true negatives (sequencing errors or germline variants). Ideally, we would like to train a classifier that predicts with high accuracy both the minority and the majority class. However, most classifiers tend to over-predict the majority class when they are trained with imbalanced data. One solution to this issue is to generate a balanced training dataset by using undersampling.

In PLATO we use undersampling to create a balanced training dataset consisting of the positive samples and an equally-sized set of likely negatives selected from the unlabeled samples. Due to the high imbalance in the unlabeled data, randomly sampling from the unlabeled samples is likely to produce a set consisting mostly of negative samples. However, some positive samples are also likely to be picked, and the randomly selected points may not be very well-separated from positive samples in the underlying feature space. Therefore our approach is to use informed undersampling, where we use the positive samples to inform the selection of likely negatives from the unlabeled set. Specifically, given sets $P$ and $U$ of positive and unlabeled samples, we generate the set $N \subseteq U$ of likely negatives as $N=\bigcup_{i=1}^{b} E_{i}$, where $\left|E_{i}\right|=|P| / b$. Each set $E_{i}$ is computed by randomly selecting a batch of $m$ samples from $U$, computing the average distance of each sample to the samples in $P$, and including in $E_{i}$ the $|P| / b$ unlabeled samples with the greatest average distance. Since the data has both categorical and numerical features, 
the Gower distance is chosen as the distance measure. We chose to generate the likely negative set $N$ by sampling multiple batches since the majority class might not be homogeneous (e.g., for SNV calling the negatives may represent sequencing errors or germline variants), and using multiple batches increases the chance of selecting representative samples from all regions of the majority class. For all experiments reported in this paper we used $b=10$ and $m=|P|$. We did not conduct extensive empirical evaluation of these choices, but reasoned that they provide a good tradeoff between having enough subsamples to give a good representation of the search space and keeping the computational costs low by avoiding too many pairwise distance computations.

For somatic variant calling, once a balanced training dataset is generated by informed undersampling, PLATO uses a random forest classifier to rank all extracted features and selects the features with above median rank. This feature selection approach falls under the category of embedded methods, and is often used to enhance generalization and reduce running time of subsequent model training. We chose to use random forestbased feature selection over alternatives such as unsupervised dimensionality reduction methods like Principal Component Analysis (PCA) since the method works well with both numeric and categorical features and retains interpretability. Random forest-based feature selection is also highly scalable. This is an important consideration in PLATO, which performs this step for multiple bootstrap samples to increase classification robustness, as detailed below. Performing feature selection independently for each bootstrap also reduces the risk of overfitting, as different sets of features may be selected for different bootstrap runs.

Due to the lower number of available features, no feature selection was performed for the MS/MS data.

\section{Bootstrapping and spy-based cutoffs}

For robustness, PLATO implements PU-learning based on informed undersampling within a bootstrapping framework and implements a scheme of automatic classification threshold selection based on spies (see the flowchart in Fig. 2). In each bootstrap iteration PLATO performs the following steps:

- Selects a set of likely negative equal to the size of $90 \%$ of positive data points using the informed undersampling method described above.

- Creates a training set by combining $90 \%$ of positive samples with the selected set of likely negatives.

- Builds a classifier using AutoML using this training set.

- Applies the classifier to the $10 \%$ of positive samples that were not included in training ("spy" samples) along with the other unlabeled samples.

- Classifies an unlabeled sample as positive, if its score is higher than the minimum score of the spy samples.

The above steps are repeated a user specified number of times ( $N$ bootstraps). A sample in $U$ is finally classified as positive if it scores higher than the spy samples in a userselected percentage of bootstrap runs, otherwise its final classification is negative. The idea of using "spies" was initially introduced in the text classification context [34]. As 
shown in the Results section, using automatically selected classification thresholds based on spies results in similar or better performance on clinical datasets than using the default classifier threshold, independent of the bootstrap support.

In general, model selection and hyperparameter tuning are complex tasks. Since exhaustively evaluating all combinations is unfeasible, we used the AutoML service integrated in Microsoft Azure to efficiently search the model space. In AutoML the user has the choice of executing an experiment on a local PC, a VM in the cloud, or a large cluster. In this work, we stored the data and executed all experiments locally. In each bootstrap of PLATO, AutoML was run on the balanced training dataset consisting of $90 \%$ of positives and an equally sized set of likely negatives generated by informed undersampling by selecting the experiment type as classification, defining the cross-validation scheme as 10-fold cross validation, and the primary metric as accuracy. For each experiment type, AutoML generates a set of initial pipeline parameters and executes a number of experiments with different parameters. In each experiment, it measures the primary metric using cross-validation and picks a new set of pipeline parameters until it reaches a threshold on execution time or the number of experiments. In the end, it builds an ensemble of different models to achieve optimal performance on the test set. Both voting and stack ensemble classifiers are currently supported. By default, they appear as the final iterations of each run. In order to have a powerful ensemble, AutoML initializes a list of up to five best scoring models (checking that their scores are within 5\% of the best score) using the Caruana et al. algorithm [35]. In subsequent iterations, a new model is added to an existing ensemble only if it improves its accuracy based on the user selected metric. The voting ensemble classifier in AutoML uses soft-voting and makes predictions based on a weighted average of predicted class probabilities. The stack ensemble classifier has a two-layer implementation. It takes the same models as the voting ensemble as the first layer, and the second layer trains a meta-model to find the optimal combination of models from the first layer. The default meta-model for classification tasks in AutoML is LogisticRegression. In our experiments, the algorithms that were run through AutoML iterations included BernoulliNaiveBayes, ExtremeRandomTrees, LightGBM, RandomForest, and SGD. However, the best-fitted models selected by AutoML were always Voting and Stack ensemble classifiers. For example, in the peptide identification problem the Voting ensemble was chosen as the best model $90 \%$ of the time and the Stack ensemble was selected $10 \%$ of the time. We have chosen 10 as the number of AutoML iterations per bootstrap based on preliminary experiments showing that more than 10 iterations yield diminishing improvements in accuracy.

\section{Processing mass-spectrometry data and assessing the false discovery rate}

RAW files were converted to MGF format using RawConverter 1.1.0.19 [36]. We used the MS-GF+ search engine [28] to search the MGF files against the human proteome, with Unspecific Cleavage, and the TDA (Target-Decoy Analysis) option turned on. The decoy database generated by MS-GF+, consisting of reversed target peptides, was concatenated with the targets for FDR estimation. The MS-GF+ MZID output files were converted to PIN (Percolator INput) format using the msgf2pin utility from Percolator. The PIN files were post-processed using Percolator as well as PLATO. Further details on MS-GF+ and Percolator settings can be found in Additional file 1. 
For estimating the false discovery rate (FDR) of peptide identifications from MS/MS data we adopted the commonly used target-decoy competition (TDC) approach. The first decision that needs to be made when using TDC is whether or not to search the target and decoy databases separately, or to concatenate them before searching. In the latter setting, each spectrum is matched with either a target or a decoy; in this way, the targets and decoys compete to match with each spectrum. As advocated in [37], we use concatenated searches in this study. Assuming that a higher score is better, for concatenated searches the FDR at a certain score threshold $t$ can be estimated as in $[29,38]$ :

$$
F D R(t)=\frac{1+\text { Number of decoy PSMs with score } \geq t}{\text { Number of target PSMs with score } \geq t}
$$

We control the FDR at level $Q$ by finding the lowest score threshold $t$ such that $F D R(t)<Q$, and only taking target PSMs with score greater than or equal to $t$. In this study, we controlled FDR at $1 \%$.

Unfortunately, controlling the FDR at a level of $\alpha$ for PSMs does not imply control at the same level for peptides. As discussed in [39], a peptide present in the sample will, on average, be matched by a greater number of spectra than an absent peptide. To address this, if a peptide matches multiple spectra, we eliminate all but the best scoring PSM for that peptide. Once we have "uniquified" the peptides, we can apply the same $q$-value cutoff procedure as for PSMs.To ensure reproducibility and fair comparisons, we created a Galaxy tool to control FDR, publicly available at neo.engr.uconn.edu/?tool_id=FDR_ custom_filter, and used it to filter the results of all compared methods (MS-GF+, Percolator, and PLATO) for which raw search results were generated as part of our empirical evaluation. More details on the precise procedure for controlling FDR at both the PSM and Peptide level as well as the Galaxy tool can be found in Additional file 1.

\section{Supplementary information}

Supplementary information accompanies this paper at https://doi.org/10.1186/s12859-020-03813-x.

Additional file 1. Supplementary Methods, Figure, and Tables.

\section{Abbreviations}

AutoML: Automated machine learning; CCCP: Consensus caller cross-platform; LightGBM: Light gradient boosting machine; MHC: Major histocompatibility complex; MS/MS: Tandem mass-spectrometry; PCA: Principal component analysis; PIN: Percolator input file; PLATO: Positive-unlabeled learning using AutoML; PPV: Positive predictive value; PSM: Peptide-spectrum match; PU: Positive-unlabeled; SGD: Stochastic gradient descent; TDA: Target-decoy analysis; TDC: Target-decoy competition; TPR: True positive rate; TRMN: Tumor-rejection mediating neoepitope.

\section{Acknowledgements}

We thank Prof. P.K. Srivastava and the members of his group at UConn Health's Carole and Ray Neag Comprehensive Cancer Center for helpful discussions and suggestions.

\section{About this supplement}

This article has been published as part of BMC Bioinformatics Volume 21 Supplement 18, 2020: Proceedings from the 8th Workshop on Computational Advances in Molecular Epidemiology (CAME 2019). The full contents of the supplement are available online at https://bmcbioinformatics.biomedcentral.com/articles/supplements/volume-21-supplement-18.

\section{Authors' contributions}

ES implemented and ran the PLATO learning method. JF ran the MS-GF+ and percolator methods. IIM designed the study and ran the CCCP pipeline. All authors read and approved the final manuscript.

\section{Funding}

Publication of this supplement is funded by the authors' institution. This work was partially supported by US Department of Education GAANN fellowships to ES and JF (Office of Postsecondary Education Grant No. P200A180092) and US 
National Science Foundation Award 1564936 to IIM. The funders had no role in study design, data collection and analysis, decision to publish, or preparation of the manuscript.

\section{Availability of data and materials}

MS/MS data analyzed in this study was downloaded from the ProteomeXchange repository using identifier PXD004894 [27]. Published Galaxy histories including runs for the $20 \mathrm{MS} / \mathrm{MS}$ melanoma datasets analyzed in this paper (grouped by patient) are available at: https://www.neo.engr.uconn.edu/u/jordan/h/bassani-mel3-public, https://www.neo.engr.uconn .edu/u/jordan/h/bassani-mel4-public, https://www.neo.engr.uconn.edu/u/jordan/h/bassani-mel5-public, https://www. neo.engr.uconn.edu/u/jordan/h/bassani-mel8-public, https://www.neo.engr.uconn.edu/u/jordan/h/bassani-mel12-publi c.

\section{Ethics approval and consent to participate}

The sequencing data analyzed in this manuscript was generated as part of the Phase I clinical trial "Study of Oncoimmunome for the Treatment of Stage III/IV Ovarian Carcinoma" (ClinicalTrials.gov Identifier: NCT02933073), conducted in accordance with FDA and Human Subject Protection regulations under IRB approval from the UConn Health Center. Written informed consent was obtained from all subjects.

\section{Consent for publication}

Not applicable.

\section{Competing interests}

ES and JF declare that they have no competing interests. IIM declares that he has a significant financial interest in Truvax Inc., a company developing personalized cancer vaccines.

Received: 5 October 2020 Accepted: 13 October 2020

Published: 30 December 2020

\section{References}

1. Schumacher TN, Schreiber RD. Neoantigens in cancer immunotherapy. Science. 2015;348(6230):69-74. https://doi. org/10.1126/science.aaa4971.

2. Srivastava PK. Neoepitopes of cancers: looking back, looking ahead. Cancer Immunol Res. 2015;3(9):969-77. https:// doi.org/10.1158/2326-6066.CIR-15-0134.

3. Castle JC, Kreiter S, Diekmann J, Löwer M, van de Roemer N, de Graaf J, Selmi A, Diken M, Boegel S, Paret C, Koslowski M, Kuhn AN, Britten CM, Huber C, Türeci Ö, Sahin U. Exploiting the mutanome for tumor vaccination. Cancer Res. 2012;72(5):1081-91. https://doi.org/10.1158/0008-5472.CAN-11-3722.

4. Duan F, Duitama J, Seesi SA, Ayres C, Corcelli S, Pawashe A, Blanchard T, McMahon D, Sidney J, Sette A, Baker B, Mandoiu II, Srivastava PK. Genomic and bioinformatic profiling of mutational neo-epitopes reveals new rules to predict anti-cancer immunogenicity. J Exp Med. 2014;211(11):2231-48.

5. Gubin MM, Zhang X, Schuster H, Caron E, Ward JP, Noguchi T, Ivanova Y, Hundal J, Arthur CD, Krebber W-J, Mulder GE, Toebes M, Vesely MD, Lam SSK, Korman AJ, Allison JP, Freeman GJ, Sharpe AH, Pearce EL, Schumacher TN, Aebersold R, Rammensee H-G, Melief CJM, Mardis ER, Gillanders WE, Artyomov MN, Schreiber RD. Checkpoint blockade cancer immunotherapy targets tumour-specific mutant antigens. Nature. 2014;515(7528):577-81. https://doi. org/10.1038/nature13988.

6. Yadav M, Jhunjhunwala S, Phung QT, Lupardus P, Tanguay J, Bumbaca S, Franci C, Cheung TK, Fritsche J, Weinschenk T, Modrusan Z, Mellman I, Lill JR, Delamarre L. Predicting immunogenic tumour mutations by combining mass spectrometry and exome sequencing. Nature. 2014;515(7528):572-6.

7. Tumor Neoantigen Selection Alliance (TESLA). https://www.parkerici.org/research-project/tumor-neoantigen-selec tion-alliance-tesla/. Accessed 24 July 2020

8. GeNeo: bioinformatics toolbox for genomics guided neoepitope prediction. https://neo.engr.uconn.edu. Accessed 24 July 2020

9. Hundal J, Carreno BM, Petti AA, Linette GP, Griffith OL, Mardis ER, Griffith M. pVAC-Seq: a genome-guided in silico approach to identifying tumor neoantigens. Genome Med. 2016;8(1):11. https://doi.org/10.1186/s1307 3-016-0264-5

10. Zhou Z, Lyu X, Wu J, Yang X, Wu S, Zhou J, Gu X, Su SZ, Chen S. TSNAD: an integrated software for cancer somatic mutation and tumour-specific neoantigen detection. R Soc Open Sci. 2017:4:170050.

11. Bjerregaard AM, Nielsen M, Hadrup SR, Szallasi Z, Eklund AC. MuPeXI: prediction of neoepitopes from tumor sequencing data. Cancer Immunol Immunother. 2017;66:1123-30.

12. Ebrahimi-Nik H, Michaux J, Corwin WL, Keller GLJ, Shcheglova T, Pak HS, Coukos G, Baker BM, Mandoiu II, BassaniSternberg M, Srivastava PK. Mass spectrometry driven exploration reveals nuances of neoepitope-driven tumor rejection. JCl Insight. 2019;4(14):e129152.

13. Krøigård $A$, Thomassen $M$, Lænkholm A-V, Kruse T, Larsen M. Evaluation of nine somatic variant callers for detection of somatic mutations in exome and targeted deep sequencing data. PLoS ONE. 2016; https://doi.org/10.1371/journ al.pone.0151664.

14. Cai L, Yuan W, Zhang Z, He L, Chou K-C. In-depth comparison of somatic point mutation callers based on different tumor next-generation sequencing depth data. Sci Rep. 2016;6:36540. https://doi.org/10.1038/srep36540.

15. Spinella J-F, Mehanna P, Vidal R, Saillour V, Cassart P, Richer C, Ouimet M, Healy J, Sinnett D. SNooPer: a machine learning-based method for somatic variant identification from low-pass next-generation sequencing. BMC Genomics. 2016;17(1):912. 
16. Fang LT, Afshar PT, Chhibber A, Mohiyuddin M, Fan Y, Mu JC, Gibeling G, Barr S, Asadi NB, Gerstein MB, et al. An ensemble approach to accurately detect somatic mutations using SomaticSeq. Genome Biol. 2015;16(1):197.

17. Fan Y, Xi L, Hughes DS, Zhang J, Zhang J, Futreal PA, Wheeler DA, Wang W. MuSE: accounting for tumor heterogeneity using a sample-specific error model improves sensitivity and specificity in mutation calling from sequencing data. Genome Biol. 2016;17(1):178,

18. Huang W, Guo YA, Muthukumar K, Baruah P, Chang MM, Skanderup AJ. SMuRF: portable and accurate ensemble prediction of somatic mutations. Bioinformatics (Oxford, England). 2019;35:3157-9.

19. Ramachandran A, Li H, Klee E, Lumetta SS, Chen D. Deep learning for better variant calling for cancer diagnosis and treatment. In: Proceedings of the 23rd Asia and South Pacific design automation conference. IEEE Press; 2018. p. 16-21. IEEE Press.

20. Sahraeian SME, Liu R, Lau B, Podesta K, Mohiyuddin M, Lam HY. Deep convolutional neural networks for accurate somatic mutation detection. Nat Commun. 2019;10(1):1041.

21. Poplin R, Chang P-C, Alexander D, Schwartz S, Colthurst T, Ku A, Newburger D, Dijamco J, Nguyen N, Afshar PT, et al. A universal SNP and small-indel variant caller using deep neural networks. Nat Biotechnol. 2018;36(10):983.

22. Ainscough BJ, Barnell EK, Ronning P, Campbell KM, Wagner AH, Fehniger TA, Dunn GP, Uppaluri R, Govindan R, Rohan TE, et al. A deep learning approach to automate refinement of somatic variant calling from cancer sequencing data. Nat Genet. 2018;50(12):1735.

23. Consensus Caller Cross-Platform (CCCP). https://neo.engr.uconn.edu/tool_runner?tool_id=CCCP. Accessed 24 July 2020

24. Duitama J, Srivastava PK, Mandoiu II. Towards accurate detection and genotyping of expressed variants from whole transcriptome sequencing data. BMC Genomics. 2012;13(Suppl 2):S6.

25. Saunders CT, Wong WSW, Swamy S, Becq J, Murray L, Cheetham RK. Strelka: accurate somatic small-variant calling from sequenced tumor-normal sample pairs. Bioinformatics. 2012;28(14):1811.

26. 2CP Filter. https://neo.engr.uconn.edu/tool_runner?tool_id=CCCP_Filter. Accessed 24 July 2020

27. Bassani-Sternberg M, Bräunlein E, Klar R, Engleitner T, Sinitcyn P, Audehm S, Straub M, Weber J, Slotta-Huspenina J, Specht K, et al. Direct identification of clinically relevant neoepitopes presented on native human melanoma tissue by mass spectrometry. Nat Commun. 2016;7:13404.

28. Kim S, Pevzner P. MS-GF+ makes progress towards a universal database search tool for proteomics. Nat Commun. 2014;5:5277. https://doi.org/10.1038/ncomms6277.

29. Käll L, Canterbury JD, Weston J, Noble WS, MacCoss MJ. Semi-supervised learning for peptide identification from shotgun proteomics datasets. Nat Methods. 2007:4(11):923.

30. Shi W, Ng CKY, Lim RS, Jiang T, Kumar S, Li X, Wali VB, Piscuoglio S, Gerstein MB, Chagpar AB, Weigelt B, Pusztai L, ReisFilho JS, Hatzis C. Reliability of whole-exome sequencing for assessing intratumor genetic heterogeneity. Cell Rep. 2018;25(6):1446-57. https://doi.org/10.1016/j.celrep.2018.10.046.

31. Garcia-Garijo A, Fajardo CA, Gros A. Determinants for neoantigen identification. Front Immunol. 2019;10:1392. https ://doi.org/10.3389/fimmu.2019.01392.

32. Sherry ST, Ward M-H, Kholodov M, Baker J, Phan L, Smigielski EM, Sirotkin K. dbSNP: the NCBI database of genetic variation. Nucleic Acids Res. 2001;29(1):308. https://doi.org/10.1093/nar/29.1.308.

33. Liaw A, Wiener M. Classification and regression by randomForest. R News. 2002;2(3):18-22.

34. Liu B, Lee WS, Yu PS, Li X. Partially supervised classification of text documents. In: ICML, vol 2. Citeseer; 2002. p. 387-394.

35. Caruana R, Niculescu-Mizil A, Crew G, Ksikes A. Ensemble selection from libraries of models. In: Proceedings of the twenty-first international conference on machine learning. 2004. p. 18.

36. He L, Diedrich J, Chu YYY, Yates JR III. Extracting accurate precursor information for tandem mass spectra by RawConverter. Anal Chem. 2015;87(22):11361-7.

37. Elias JE, Gygi SP. Target-decoy search strategy for increased confidence in large-scale protein identifications by mass spectrometry. Nat Methods. 2007;4(3):207.

38. Kall L. Email communication. (2019).

39. Granholm V, Navarro JF, Noble WS, Käll L. Determining the calibration of confidence estimation procedures for unique peptides in shotgun proteomics. J. Proteomics. 2013;80:123-31. https://doi.org/10.1016/j.jprot.2012.12.007.

\section{Publisher's Note}

Springer Nature remains neutral with regard to jurisdictional claims in published maps and institutional affiliations.

Ready to submit your research? Choose BMC and benefit from:

- fast, convenient online submission

- thorough peer review by experienced researchers in your field

- rapid publication on acceptance

- support for research data, including large and complex data types

- gold Open Access which fosters wider collaboration and increased citations

- maximum visibility for your research: over 100M website views per year

At BMC, research is always in progress.

Learn more biomedcentral.com/submissions 